\begin{tabular}{|lrlll} 
September 28, & 2018 & $4: 42$ & WSPC/INSTRUCTION & FILE
\end{tabular} consistency accepted

(2018 4:42 $\mathrm{WSPC}$ INSTRUCTION

Modern Physics Letters A

(C) World Scientific Publishing Company

\title{
Consistency Condition of Spherically Symmetric Solutions in $f(R)$ Gravity
}

\author{
Reza Saffari \\ Institute for Advanced Studies in Basic Sciences, P.O. Box 45195-1159, Zanjan, Iran, * \\ Sohrab Rahvar \\ Department of Physics, Sharif University of Technology, P.O.Box 11365-9161, Tehran, Iran, ${ }^{\dagger}$
}

Received (Day Month Year)

Revised (Day Month Year)

\begin{abstract}
In this work we study the spherical symmetric solutions of $f(R)$ gravity in the metric formalism. We show that for a generic $f(R)$ gravity, the spherical symmetric solution is consistent with the modified gravity equations except in the case of imposing an extra condition for the metric.
\end{abstract}

Keywords: Cosmology; Dark Energy; Modified Gravity.

95.36.+x, 98.80.Jk, 98.80.Es

*rsaffari@iasbs.ac.ir

${ }^{\dagger}$ rahvar@sharif.edu 
\begin{tabular}{|lllll} 
September 28, & 2018 & $4: 42$ & WSPC/INSTRUCTION & FILE
\end{tabular}

2 Saffari \& Rahvar

Recent observations of the Supernova Type Ia and Cosmic Microwave Background $(\mathrm{CMB})$ radiation indicate that universe is under positive accelerating expansion $1,2,3,4$. One of the possible solutions to explain the dynamics of universe is replacing modified gravity models with the Einstein-Hilbert action $5,6,7,20,9,10,11$. The simplest class of this models is using a generic action of $f(R)$ instead of $R$ in the action. The aim regarding $f(R)$ gravity models is that, these models can cover all the domains from the cosmological to the solar system scales. In the cosmological scales some of the models not only can provide a late time acceleration for the universe but also predict an inflationary phase for the early universe ${ }^{12}$. In the solar system scales, there is a long list of works in the spherically symmetric solution of $f(R)$ gravity both in the metric and the Palatini formalisms $13,14,15,16,17,18,19,20,21,22,23,24,25,26,27,28,29$. In the Palatini formalism the solution of vacuum space is a Schwarzschild-de'Sitter metric with an effective cosmological constant obtain from the vacuum solution of $f(R)$ gravity.

In the metric formalism recent attempts is using the inverse method to derive the solar system and galactic scale dynamics. However we should note that the solution may not satisfy the field equations if we impose an extra condition on the metric elements $26,27,28,29$. Here in this work we revisit the spherically symmetric solutions of modified gravity in the metric formalism with more details and present the missed points in the recent literatures.

We start with a generic form of metric for the spherically symmetric space:

$$
d s^{2}=-B(r) d t^{2}+\frac{X(r)}{B(r)} d r^{2}+r^{2}\left(d \theta^{2}+\sin ^{2} \theta d \phi^{2}\right)
$$

For a generic form of Lagrangian as a function of Ricci scalar, the action is written as:

$$
S=\frac{1}{2 \kappa} \int d^{4} x \sqrt{-g} f(R)+S_{m}
$$

Varying action with respect to the metric results in the field equation as:

$$
F(R) R_{\mu \nu}-\frac{1}{2} f(R) g_{\mu \nu}-\left(\nabla_{\mu} \nabla_{\nu}-g_{\mu \nu} \square\right) F(R)=\kappa T_{\mu \nu},
$$

where $F=d f / d R$ and $\square \equiv g^{\mu \nu} \nabla_{\mu} \nabla_{\nu}$. From equation (3), we take trace and obtain action in terms of $f, F$ and Ricci scalar

$$
f(R)=\frac{1}{2}(3 \square F+F R-\kappa T) .
$$

Substituting $f(R)$ from equation (4) in (3), field equation obtain as:

$$
R_{\mu \nu}-\frac{1}{4} g_{\mu \nu} R=\frac{\kappa}{F}\left(T_{\mu \nu}-\frac{1}{4} g_{\mu \nu} T\right)+\frac{1}{F}\left(\nabla_{\mu} \nabla_{\nu} F-\frac{1}{4} g_{\mu \nu} \square F\right) .
$$

This equation is diagonal, depends only on $r$. For simplicity in calculation we rewrite Eq. (5) as:

$$
K_{[\mu]}=\frac{F R_{\mu \mu}-\nabla_{\mu} \nabla_{\mu} F-\kappa T_{\mu \mu}}{g_{\mu \mu}}
$$


September 28, $2018 \quad 4: 42$ WSPC/INSTRUCTION $\quad$ FILE

Consistency Condition of Spherically Symmetric Solutions in $f(R)$ Gravity 3

where $K_{[\mu]}$ is an index independent parameter. Here the right hand side of $\mu=$ $t, r, \theta$ terms except $\mu=\phi$, are independent and the field equation reduces to three independent equation. On the other hand the constrain of $K_{[t]}=K_{[r]}=K_{[\theta]}$, reduces the number of independent equations to two. For the vacuum space $T_{\mu \nu}=0$, $K_{[t]}-K_{[r]}=0$ results in:

$$
\frac{X^{\prime}}{X}=\frac{2 r F^{\prime \prime}}{2 F+r F^{\prime}}
$$

and from $K_{[t]}-K_{[\theta]}=0$,

$$
B^{\prime \prime}+\left(\frac{F^{\prime}}{F}-\frac{1}{2} \frac{X^{\prime}}{X}\right) B^{\prime}-\frac{2}{r}\left(\frac{F^{\prime}}{F}-\frac{1}{2} \frac{X^{\prime}}{X}\right) B-\frac{2}{r^{2}} B+\frac{2}{r^{2}} X=0 .
$$

We note that since we are working in vacuum space, the generalized Bianchi identity provides no more extra constrain on the field equations.

Now our aim is to extract $B$ and $X$ as the metric elements from equations (7) and (8). These two differential equations contain one more function of $F(r)$. This means that to have a unique solution for the metric we have to fix either one of the metric elements (i.e. $X, B$ ) or the action $F$. We study both cases. Fist let us start with fixing one of the metric elements.

Fixing metric elements: Let us assume fixing $X(r)$. In this case $F(r)$ can be obtained from the equation (7) and substituting this term and $X(r)$ in equation (8) we can obtain $B(r)$. The same procedure can be applied for the case of fixing $B(r)$. From the metric elements one can extract the Ricci scalar in terms of $r$. Eliminating $r$ in favor of $R$ in the expression, $F(r)$ we can obtain the action as a function of Ricci scalar. This approach is so-called inverse problem, means that knowing the dynamics of a test particle in the spherically symmetry space we can demand for a proper action for the gravity ${ }^{23,24,25,26}$. As a simple example let us choose $X=1$. In this case the equation ( 7$)$ reduce to $F^{\prime \prime}=0$ and the solution is

$$
F(r)=a+b r .
$$

Now substituting this action and $X=1$ in equation (8) results in a differential equation for $B$ :

$$
B^{\prime \prime}-\frac{2}{r^{2}} B+\frac{2}{r^{2}}+\frac{b}{a+b r}\left(B^{\prime}-\frac{2}{r} B\right)=0,
$$

The exact solution of this differential equation is:

$$
\begin{aligned}
B(r)= & 1-\frac{b}{a} r+\left(\frac{3}{2}+\ln \left|\frac{b}{a}+\frac{1}{r}\right|\right) \frac{b^{2}}{a^{2}} r^{2}+C_{1} r^{2} \\
& -C_{2}\left[\frac{1}{3 r}-\frac{b}{2 a}+\frac{b^{2}}{a^{2}} r-\frac{b^{3}}{a^{3}} r^{2} \ln \left|\frac{b}{a}+\frac{1}{r}\right|\right] .
\end{aligned}
$$

For the case of $b=0$, equation (9) reduces to the Einstein-Hilbert action and from the equation (11) we recover the Schwarzschild-de'Sitter metric. 
Now we seek the other special solution, choosing $a=0$. In this case the following solution obtain for the metric from the equation (10):

$$
B(r)=\frac{1}{2}+C_{1} r^{2}+C_{2} r^{-2},
$$

The corresponding Ricci scalar of this metric is:

$$
R=\frac{1}{r^{2}}-12 C_{1} \text {. }
$$

Substituting this equation in $F(r)=b r$ and integrating it results in the action in terms of Ricci scalar:

$$
f(R)=2 b \sqrt{R+12 C_{1}}+C_{3} .
$$

For the generic case where $a, b \neq 0$, action can only be calculated numerically.

Depending on our desired dynamics in the spherically symmetric space we can calculate proper action by this method. For instance one can use the dynamics of stars in the galaxy to replace the dark matter with a proper action ${ }^{11,23,25}$. For the case of choosing a complicated function for $X(r)$, the equations (7) and (8) should be calculated numerically.

Action fixing: The second approach is fixing action $f(R)$, means that for a given action, we want to obtain the metric elements, $X$ and $B$. We substitute the action in the equations (7) and (8) where the Ricci scalar is expressed in terms of the metric elements as follows:

$$
R=-\frac{1}{X}\left[B^{\prime \prime}+\frac{4}{r} B^{\prime}+\frac{2}{r^{2}} B-\frac{X^{\prime}}{X}\left(\frac{1}{2} B^{\prime}+\frac{2}{r} B\right)\right]+\frac{2}{r^{2}},
$$

We can determine the metric elements by the numerical solution of the differential equations (7) and (8). An important point in this procedure is that putting an extra condition for the metric elements may cause inconsistent solutions ${ }^{26,27,28,29}$. The reason is very simple, adding one more constrain will decrease the number of parameters to one, while we have two independent differential equations of (7) and (8). For instance let us choose $X=1$ constrain on the metric elements. From equation (15) we can obtain $B(r)$ in terms of Ricci scalar as follows:

$$
B(r)=1+\frac{C_{1}}{r}+\frac{C_{2}}{r^{2}}-\frac{1}{r^{2}} \int\left[\int r^{2} R(r) d r\right] d r,
$$

where $R(r)$ can be obtained from $F^{\prime \prime}(r)=0$. We rewrite this equation after changing the differentiation to the Ricci scalar as follows

$$
R^{\prime \prime} f^{(2)}(R)+R^{\prime 2} f^{(3)}(R)=0,
$$

where $f^{(n)}(R) \equiv d^{n} f / d R^{n}$. For a given $f(R)$, we can obtain the dependence of the Ricci scalar to the radial distance. Comparing $B(r)$ in equation (16) with that of (11) shows that in general those two equations are not identical. In another word the metric element in equation (16) is an inconsistent solution for the spherically symmetric space. 
\begin{tabular}{|lllll} 
September 28, & $2018 \quad 4: 42$ & WSPC/INSTRUCTION & FILE
\end{tabular}

Consistency Condition of Spherically Symmetric Solutions in $f(R)$ Gravity 5

Summarizing this letter, we pointed out that the modified gravity equations in the form of $f(R)$, reduce to two independent differential equations in the spherically symmetric space. These equations contains $X, B$ as the metric elements and $F$ as the derivative of the action. The metric elements as well as the action can be obtained by fixing one of the them. Fixing $X$ or $B$, so-called the inverse problem in $f(R)$ gravity means that we can derive a proper action if we know the dynamics in the spherically symmetric space. In the second approach one can introduce the action and calculate the metric elements. Finally we emphasis on considering extra conditions for the metric, which results inconsistent solutions for the space. This point is missed in some of the literatures that try to replace the dark matter with the modified gravity in the Galactic scales.

\section{References}

1. G. Hinshaw et al., Astrophys. J. Suppl. 170, 288 (2007).

2. D. N. Spergel et al., Astrophys. J. Suppl. 170, 377 (2007).

3. S. Perlmutter et al. (Supernova Cosmology Project Collaboration), Astrophys. J. $\mathbf{5 1 7}, 565$ (1999).

4. A. G. Riess et al. (Supernova Search Team Collaboration), Astron. J. 116, 1009 (1998).

5. S. M. Carroll, V. Duvvuri, M. Trodden, and M. S. Turner, Phys. Rev. D 70, 043528 (2004).

6. S. Nojiri and S. D. Odintsov, Phys. Rev. D 68, 123512 (2003).

7. S. Nojiri and S. D. Odintsov, Gen. Rel. Grav. 36, 1765 (2004).

8. G. J. Olmo, Phys. Rev. D 72, 083505 (2005).

9. S. Baghram, M. Farhang, and S. Rahvar, Phys. Rev. D 75, 044024 (2007).

10. M. S. Movahed, S. Baghram, and S. Rahvar Phys. Rev. D 76, 044008 (2007).

11. S. Rahvar, Y. Sobouti, (accepted in Mod. Phys. Lett. A) astro-ph/0704.0680 (2008).

12. T. P. Sotiriou Phys. Rev. D 73063515 (2006).

13. I. Navarro and K. V. Acoleyen, Phys. Lett. B 622, 1 (2005).

14. T. Clifton and J. D. Borrow, Phys.Rev. D 72, 103005 (2005).

15. T. Clifton, Class. Quant. Grav. 237445 (2006).

16. A. L. Erickcek, T. L. Smith, and M. Kamionkowski, Phys.Rev. D 74, 121501 (2006).

17. G. Allemandi and M. L. Ruggiero, Gen. Rel. Grav 39, 1381 (2007).

18. I. Navarro and K. V. Acoleyen, JCAP 0702, 022 (2007).

19. T. Chiba, T. L. Smith and A. L. Erickcek, Phys.Rev. D 75, 124014 (2007).

20. G. J. Olmo, Phys. Rev. Lett. 98, 061101 (2007).

21. Gonzalo J. Olmo, Phys.Rev. D 75, 023511 (2007).

22. M. D. Seifert, Phys. Rev. D 76, 064002 (2007).

23. Y. Sobouti, A\&A 464, 921 (2007).

24. R. Saffari and Y. Sobouti, A\&A 472, 833 (2007).

25. R. Saffari and S. Rahvar, Phys. Rev. D 77, 104028 (2008).

26. T. Multamäki and I. Vilja, Phys. Rev. D 74, 064022 (2006).

27. S. Capozziello, V. F. Cardone and A. Troisi, MNRAS, 375, 1423 (2007).

28. S. Capozziello and M. Francaviglia, astro-ph/0706.1146.

29. S. Capozziello, A. Stabile and A. Troisi, gr-qc/0709.0891. 